\title{
Transposition
}

Musique et Sciences Sociales

$8 \mid 2019$

Musique : patrimoine immatériel ?

\section{Patrizia Veroli, Gianfranco Vinay (eds.), Music- Dance. Sound and Motion in Contemporary Discourse}

London \& New York, Routledge, 2018

\section{Sophie Jacotot}

\section{(2) OpenEdition}

\section{Journals}

Édition électronique

URL : http://journals.openedition.org/transposition/2975

DOI : $10.4000 /$ transposition.2975

ISSN : 2110-6134

Éditeur

CRAL - Centre de recherche sur les arts et le langage

Référence électronique

Sophie Jacotot, « Patrizia Veroli, Gianfranco Vinay (eds.), Music-Dance. Sound and Motion in Contemporary Discourse », Transposition [En ligne], 8 | 2019, mis en ligne le 15 septembre 2019, consulté le 17 décembre 2020. URL : http://journals.openedition.org/transposition/2975 ; DOI : https://doi.org/10.4000/transposition.2975

Ce document a été généré automatiquement le 17 décembre 2020.

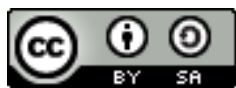

La revue Transposition est mise à disposition selon les termes de la Licence Creative Commons Attribution - Partage dans les Mêmes Conditions 4.0 International. 


\section{Patrizia Veroli, Gianfranco Vinay (eds.), Music-Dance. Sound and Motion in Contemporary Discourse}

London \& New York, Routledge, 2018

Sophie Jacotot

\section{RÉFÉRENCE}

Patrizia Veroli, Gianfranco Vinay (eds.), Music-Dance. Sound and Motion in Contemporary

Discourse, London \& New York, Routledge, 2018, 268 p.

1 Patrizia Veroli et Gianfranco Vinay placent leur publication Music-Dance. Sound and Motion in Contemporary Discourse sous le signe de la «complexité ", notion empruntée à Edgar Morin et dont la congruence, pour une approche théorique de la musique et de la danse, naît de plusieurs constats : le caractère inséparable du son et du mouvement dans la performance dansée et sa réception; l'implication du corps de l'auditeur/trice dans l'expérience musicale et dans la construction de sens relative à une performance. Dans sa préface, Gianmario Borio, directeur de la Fondation Cini à Venise, situe le contexte d'émergence de ce livre, soit un groupe de chercheurs/ses rassemblé.e.s pour le colloque organisé en 2015 à l'occasion du $30^{\mathrm{e}}$ anniversaire de l'Institut de Musique de la Fondation Cini. Cet événement fut l'occasion de faire le point sur les collaborations interdisciplinaires et les enrichissements méthodologiques réciproques entre musicologie et études en danse avec comme point d'intérêt commun la rencontre entre mouvements et sons.

2 Le livre porte sur le temps long $d u x^{e}$ siècle, riche de collaborations entre compositeurs et chorégraphes. Les plus célèbres comme celles de Balanchine et Stravinsky, Cage et Cunningham, sont présentes dans l'ouvrage, mais celui-ci s'aventure le plus souvent sur des territoires moins connus, comme les pratiques notationnelles des chorégraphes, les tentatives chez les compositeurs de trouver une 
représentation graphique du geste musical, ou encore la prise en compte de l'expérience dansée comme paradigme d'une perception multimodale. Le terme clé, bien qu'il n'apparaisse pas dans le titre, est celui de "choreomusicology ", que l'on peut traduire par " choréomusicologie » ou «études choréomusicales", dont le dessein est d'appréhender conjointement danse et musique, ainsi que leurs relations. L'objet de l'introduction rédigée par Patrizia Veroli et Gianfranco Vinay est précisément de faire le point sur la signification de ce terme relativement récent dans les publications en anglais puisqu'il apparait en 1992 dans un ouvrage de Paul Hodgins' ${ }^{1}$. Reflet de l'état de l'art actuel, l'ouvrage réunit quinze auteurs/autrices provenant d'horizons variés : choréomusicologues, historien.ne.s de la danse, musicologues, spécialistes des sciences cognitives et des performance studies.

3 La première partie de l'ouvrage rassemble des textes qui questionnent les fondements théoriques de la choréomusicologie. Inger Damsholt, l'une des pionnières dans les études choréomusicales, revient dans le premier chapitre sur le développement de ce champ disciplinaire depuis 25 ans et sur les tensions, entre pratique artistique et recherche académique, ou encore entre approche formaliste et approche culturaliste, qui ont marqué son historiographie. Elle souligne la difficulté qui demeure à le circonscrire, la choréomusicologie pouvant apparaître, selon les auteur.e.s, comme un champ de recherche à part entière, ou bien comme un terrain privilégié pour une approche interdisciplinaire de la performance. Dans le second chapitre, Massimiliano Locanto prône une choréomusicologie au-delà du "formalisme", tout en se revendiquant héritier d'une tradition musicologique soucieuse de l'analyse des partitions. Il y parvient en utilisant le concept de « geste musical »- tel que défini par Robert Hatten ${ }^{2}$, tant dans sa dimension métaphorique que dans sa dimension intermodale. Il illustre son propos par l'étude de la pièce Variations for Orchestra, dernier ballet de Balanchine (1982), composé sur la musique de Stravinsky, Variations Aldous Huxley in Memoriam (1963-1964), en mettant en regard la partition musicale et l'analyse de séquences de mouvements à partir d'une captation vidéo de la danse. Lawrence $\mathrm{M}$. Zbikowski analyse quant à lui des extraits des Noces (1923) de Bronislava Nijinska, dans la version remontée par la chorégraphe elle-même en 1966 pour le Royal Ballet, et filmée par Ross MacGibbon en 2001 pour la BBC. Il s'intéresse à la connexion entre les mouvements dansés et la musique de Stravinsky, soulignant tout à la fois la synchronisation entre musique et danse et les passages où leur dialogue s'enrichit et se complexifie - les danseurs proposant par exemple une ligne rythmique supplémentaire. À travers cette étude de cas, l'auteur questionne plus largement la manière dont l'expérience corporelle et les informations proprioceptives configurent nos processus cognitifs, permettant d'envisager autrement la compréhension du fait musical. Pour clore cette première partie, Stephanie Jordan - pionnière elle aussi dans le champ des études choréomusicales ${ }^{3}$ - étudie, après un survol historique des rapports entre compositeurs et chorégraphes depuis le XIX ${ }^{\mathrm{e}}$ siècle, le cas du chorégraphe Mark Morris lorsqu'il utilise pour ses créations des musiques écrites préalablement. Elle explore la diversité des approches choréomusicales utilisées par Morris dans ses pièces entre les années 1980 et le début des années 2000, depuis la «visualisation musicale » à la façon de Ruth Saint-Denis et Doris Humphrey, jusqu'à une approche quasicunninghamienne de la matière musicale faisant office de paysage sonore.

4 La seconde partie aborde la question de la notation musicale et de la "choré-graphie » dans son sens premier, c'est-à-dire des systèmes graphiques d'écriture du mouvement. Claudia Jeschke analyse les mutations des systèmes de notation du mouvement depuis 
le XIX ${ }^{e}$ siècle, notamment dans ce qu'ils mettent en lumière des rapports entre musique et danse. Elle souligne que, depuis les dernières décennies du $\mathrm{xx}^{\mathrm{e}}$ siècle, les notations ne sont plus seulement des moyens de consigner et de conserver la danse, mais deviennent des outils multifonctionnels, impliqués de façon nouvelle dans les processus perceptifs et faisant l'objet d'usages performatifs inédits. Marina Nordera rappelle quant à elle que les notations du mouvement opèrent une synthèse visuelle, dans des signes graphiques, de l'acte dansé, lequel engage une perception multimodale (visuelle, auditive, tactile, kinesthésique, proprioceptive). En s'appuyant sur l'analyse de systèmes anciens datant $\mathrm{du} \mathrm{xv}^{\mathrm{e}}$ siècle et de la Chorégraphie de Raoul-Auger Feuillet (1700), elle propose de conférer un statut épistémologique aux notations dans la mesure où elles font écho aux techniques corporelles et aux savoirs pratiques de leur temps. Elle évoque aussi des usages récents de notations conçues comme outils de composition par des chorégraphes de danse contemporaine, comme Laurence Pagès et Christina Towle utilisant des partitions de souffle. Après avoir rappelé la présence tacite du corps dans le système d'écriture musical traditionnel, Nicolas Donin étudie le cas de compositeurs (Helmut Lachenmann, Vinko Globokar...) de la seconde moitié du $\mathrm{xx}^{\mathrm{e}}$ siècle qui ont inventé de nouveaux systèmes de notation dans lesquels le corps occupe une place originale. L'attention aux gestes des musiciens, à leur souffle, à leur posture, devient alors centrale, allant jusqu'à la disparition des instruments et rejoignant de ce fait des problématiques chorégraphiques.

5 La troisième partie de l'ouvrage porte sur les négociations autour de la question de l'auctorialité entre musique et danse. Julia $\mathrm{H}$. Schröder trace une typologie des relations choréomusicales depuis les années 1950, de l'indépendance prônée par John Cage et Merce Cunnigham, jusqu'à l'utilisation du geste musical comme matériau chorégraphique par Xavier Le Roy, en passant par l'analogie structurelle entre la musique de Thierry de Mey et la danse d'Anne Teresa de Keersmaeker. Pour étudier des musicien.ne.s qui performent une partition en silence, ou des performers qui dansent sur une musique différente de ce qu'entend le public, elle convoque les notions de transmédialité et d'intermédialitét. Ulrich Mosch étudie le ballet Raramente (1971) dont la danse a été composée par Aurel Miloss, chorégraphe alors au faîte de sa carrière, tandis que le compositeur Sylvano Bussotti signait le livret, la musique, les lumières, les costumes et les décors. La relation asymétrique entre les deux protagonistes est analysée à partir des multiples sources relatives à cette œuvre: partition musicale, "script ", maquettes de costumes et décors, captation, réception critique. Susanne Franco se penche quant à elle sur la pièce à succès du chorégraphe Christian Rizzo, D'après une histoire vraie (2013), dans un contexte où la danse contemporaine renoue avec la notion de "répertoire " en proposant des relectures distanciées de danses traditionnelles ou de danses du passé. Elle décèle une vision exotisante dans l'approche de Rizzo, s'inspirant librement de danses masculines du pourtour méditerranéen, comme dans celle des deux batteurs Didier Ambact et King Q4, auteurs de la musique et présents au plateau.

6 La quatrième partie s'intitule «Corps sentant » et questionne la rencontre entre le son et le mouvement au sens où elle déclenche des émotions et des formes spécifiques de cognition. Eric F. Clarke explore ainsi les principes psychologiques et physiologiques permettant de comprendre les expériences et les réactions des auditeurs/auditrices et spectateurs/spectatrices face au son et au mouvement. Il éclaire le lien entre perception et action à l'aune des recherches en sciences cognitives sur l'empathie et les 
neurones-miroirs, et évoque la plénitude sensorielle qui brouille la distinction entre objet et sujet. Il prend l'exemple du moonwalk de Michael Jackson et du danseur de popping Marquese Scott pour analyser la relation entre les mouvements du corps et ce qu'il appelle des musi-mouvements ou mouvements induits par la musique. Rolf Inge Godøy rappelle quant à lui la reconnaissance, depuis deux décennies, du caractère multimodal de l'expérience musicale dans la mesure où sa perception mêle différents types de sensations, outre la perception du son proprement dit: perception du mouvement produisant le son, empathie kinesthésique... Il souligne combien les nouvelles technologies permettent d'aller plus loin dans l'analyse de la relation entre son et mouvement en proposant la notion de "motormimetic» ou de cognition contagion? - «motrice mimétique ». Dee Reynolds explore la relation synesthésique entre la vue, l'ouïe et le toucher, à travers l'exemple de l'installation See, Sea: An exploration of memory and time (2013) de la chorégraphe Susan Sentler, comprenant des films, des photographies, de la danse et de la musique. En référence au système de l'Effort de Laban et aux qualités dynamiques qui découlent, selon la terminologie labanienne, de l'usage du poids, de l'espace, du temps et du flux, elle analyse notamment la manière dont les images (visuelles et sonores) de l'eau et de la mer mettent en jeu le concept de perception haptique, impliquant des sensations tactiles. Susan Broadhurst adopte une double perspective pour analyser le lien entre performances contemporaines et nouvelles technologies : celle de la phénoménologie d'une part et celle, d'autre part, de la neuro-esthétique, nouveau champ de recherche qui engage un dialogue entre les neurosciences, la science informatique et l'étude des actes créatifs et de leur réception. Antonio Camurri s'intéresse dans le dernier chapitre à la manière dont la science et l'art peuvent mutuellement s'enrichir aujourd'hui en présentant différents projets qui réunissent scientifiques et artistes, dans les domaines de la musique et de la danse. Le développement de modèles computationnels (au croisement des sciences cognitives, de la psychologie, de l'informatique et des mathématiques) permet ainsi d'analyser des phénomènes comme les interactions émotionnelles des performers entre eux ou la perception des qualités de mouvement par les spectateurs/spectatrices, permettant des développements originaux sur la notion d'expressivité.

7 Véritable somme, l'ouvrage dirigé par Patrizia Veroli et Gianfranco Vinay ouvre de multiples pistes de réflexion sur les rapports entre danse et musique. La confluence d'approches historiques et esthétiques, l'apport des neurosciences, la considération de la performance musico-chorégraphique dans son entièreté, sont autant d'éléments qui permettent d'envisager de nouvelles explorations et appellent de nouvelles collaborations - choréomusicologiques - entre chercheur.se.s en danse et chercheur.se.s en musique, à l'instar de ce qui se produit couramment entre danseur.se.s et musicien.ne.s, entre chorégraphes et compositeurs/trices. 


\section{NOTES}

1. HODGINS Paul, Relations Between Score and Choreography in Twentieth-Century Dance: Music, Movement and Metaphor, London, Mellen, 1992.

2. HATTEN Robert S., Interpreting Musical Gestures, Topics, and Tropes: Mozart, Beethoven, Schubert, Bloomington, Indiana University Press, 2004.

3. JORDAN Stephanie, "Choreomusical Conversations: Facing a Double Challenge ", Dance Research Journal, vol. 43, $\mathrm{n}^{\circ}$ 1, 2011, p. 43-64.

4. MÜLLER Jürgen E., «L'intermédialité, une nouvelle approche interdisciplinaire: perspectives théoriques et pratiques à l'exemple de la vision de la télévision ", Cinémas, vol. 10, n 2-3, 2000, p. 105-134.

\section{AUTEURS}

\section{SOPHIE JACOTOT}

Danseuse et historienne de la danse. Docteure en histoire de l'Universitẻ Paris 1, spécialiste de l'histoire du bal à l'époque contemporaine, elle publie en 2013 Danser à Paris dans l'entre-deuxguerres. Lieux, pratiques et imaginaires des danses de société des Amériques (1919-1939). Chercheuse associée au Centre d'histoire sociale $\mathrm{du} \mathrm{xx}^{\mathrm{e}}$ siècle, elle s'intéresse aux systèmes de notation $\mathrm{du}$ mouvement, se forme en écriture Conté avec Michelle Nadal et Catherine Augé, puis en cinétographie Laban au CNSMDP avec Noëlle Simonet. Depuis 2008, elle travaille auprès de la chorégraphe Dominique Brun, comme chercheuse pour le film Chanel et Stravinsky de Jan Kounen, puis comme assistante et interprète pour ses pièces Sacre \# 2 (2014), Jeux. Trois études pour sept petits paysages aveugles (2017) et, bientôt, Noces (création 2020). 